\title{
Feature of Statistical Projection Algorithm-based Image Retrieval
}

\author{
Xiaofei Zheng ${ }^{1, *}$, Zhe Gao ${ }^{2}$ and Wei Luo ${ }^{1}$ \\ ${ }^{1}$ Institute of Scientific and Technical Information. CATAS, 571737, Danzhou Hainan, China \\ ${ }^{2}$ Editorial Department of Journal, Hainan University, Haikou 570228, China
}

Received: 3 Apr. 2013, Revised: 4 Aug. 2013, Accepted: 6 Aug. 2013

Published online: 1 Mar. 2014

\begin{abstract}
In this paper, image retrieval method based on statistics and projection is presented. At first, images are transferred from the RGB color model to the HSI color model, then images are segmented into sub-images (or blocks) and extract the main hue and main intensity in these sub-images. Images' main hue and main intensity are projected onto the horizontal and vertical directions, respectively, and four projective histograms were obtained. Finally, the first three central moments are taken as feature as to compute the similarity between images.
\end{abstract}

Keywords: main hue, main intensity, projective histogram

\section{Introduction}

Content-based image retrieval (Content-based Image Retrieval, referred to as CBIR) is the use of visual features of the image, such as color, texture, shape, match the query image and database, return similar images to the user. Color is the most significant feature of the visual image, is also the main perceptual features of people to identify the image, and has received extensive attention and research of [1] in image retrieval. Swain and Ballard [2] proposed the global color histogram, histogram intersection - L1 metric method, [2] color histogram as the similarity measure. Color histogram is the statistics of pixel in an image shows the times, can only reflect the frequency of different color image value, can not reflect the distribution of the relationship between color space, the image retrieval accuracy is not high.

In order to overcome the defects of the histogram distribution in space, this paper proposes image retrieval algorithm based on statistical projection. Firstly, the image is transformed from RGB model to HSI model, extracting principal value (primary color (Main Hue) [3] and the main gray (Main Intensity), the main color and gray projection in horizontal and vertical directions, get projection histogram [4], calculate the similarity of the images of the first three moments using projection histogram.

\section{Retrieval Algorithm Based on Statistical Projection Method}

\subsection{Colour Model}

Search in the use of color image, select the appropriate color model is especially important for search results. Commonly used color model are: RGB color model, namely the use of R (red), G (green), B (blue) color to represent the color, RGB color model is widely used in display system, but the visual perception of RGB color model and people do not correspond, similar in color similar to that of the RGB model does not reflect the color itself, in the use of RGB color model to retrieve images are RGB color model conversion to other color space.

Because the visual system of HSI color model with the most close to, is a suitable for human resolution model [4]. In HIS model, $\mathrm{H}$ said the tone (Hue), S represents the color saturation (Saturation), I denote brightness or intensity (Intensity). For any $3 \mathrm{R}, \mathrm{G}$, and B value in the range of $[0,1]$, can be converted to [5] HSI color model by the following formula:

$$
\begin{gathered}
I=\frac{1}{3}(R+G+B), \\
S=1-\frac{3}{R+G+B} \min (R, G, B),
\end{gathered}
$$

\footnotetext{
*Corresponding author e-mail: 615744175@qq.com
} 


$$
H= \begin{cases}\arccos \frac{(R-G)+(R-B)}{2 \sqrt{(R-G)^{2}+(R-B)(G-B)}}, & G \geq B \\ 2 \pi-\arccos \frac{(R-G)+(R-B)}{2 \sqrt{(R-G)^{2}+(R-B)(G-B)}}, & G<B .\end{cases}
$$

The range of calculated and formula for $[0,1]$, in the range of $[0,2 \pi]$, in order to make also in $[0,1]$, order $=H=$ $H /(2 \pi)$ conversion.

\subsection{The Main Value Extraction}

The system using the algorithm, fixed block image segmentation method. Firstly, the block is fixed, the image.

Divided into same size grid, in order to describe color feature and gray feature of each block, this paper adopts the concept of value.

Definition (main) for a characteristic block value (color or gray value), if it has the most number of pixels in the block, then the characteristic values (color or gray value) is defined as the block principal value. Let be the first block main value, then:

$$
M_{i}=\left\{k \mid \operatorname{num}(i, k)=\max _{j=1}^{256}(\operatorname{num}(i, j))\right\}, 1 \leq k \leq 256
$$

Among them, said the tone value (or gray), said the tone of the block number of values of all pixels for the.

However, when the block is the image to the principal value is not may reflect the color feature and shape feature block, if the extracted principal value in the whole block contains a very small proportion of the value, main value is extracted is not enough to reflect the characteristics of the block, in order to eliminate the effect of this case the main value judgment, the following strategy to determine the extracted principal value as the block is class:

1.The set threshold (threshold 1$)$, if num $(i, k) /(m \times n) \geq$ threshold $_{1}, M_{i}=k$ is taken as the block principal value. 2.if $\operatorname{num}(i, k) /(m \times n)<$ threshold $_{1}$, you need to consider the block near the block's main value to determine the block principal value, if the principal value of the adjacent block satisfy condition (1) and the block in the main value is equal to or less than a certain (threshold) of the difference, $M_{i}=k$ is still set to the principal value, otherwise do not specify the main block. Among them, $m, n$ said, every block size.

\subsection{Projection Histogram}

For a certain image features (such as component or components), with characteristics of the histogram, the histogram is a discrete function, i.e.:

$$
H(k)=\frac{n_{k}}{N}, k=0,1, \ldots, L-1
$$

Type: characteristics of representative value of the image, are the number of feature values, are the number of pixels having characteristic values for the image, and are the total number of the image pixels.

Since the histogram is each pixel in an image of a statistical characteristic value of the frequency of occurrence of, only reflect the frequency of one image feature, spatial relationship without inclusion of image features, is applied to image retrieval, will reduce the accuracy of retrieval system.

In order to overcome the histogram does not reflect the image of a characteristic spatial distribution, this method in the statistical foundation block principal value using projection histogram. One feature of an image of each pixel are projected on the horizontal axis and the vertical axis, get the distribution of the features in the two directions. Projection component in the direction and direction, reflects the distribution of the image color space; projection component in the direction and direction, reflects the shape of the image.

For an image of the component in the direction and the direction of the projection histogram is defined as follows:

$$
\begin{aligned}
& P_{H}(x)=\frac{1}{h} \sum_{y=1}^{h} H(x, y), x=1,2, \ldots, w \\
& P_{H}(y)=\frac{1}{w} \sum_{y=1}^{w} H(x, y), y=1,2, \ldots, h .
\end{aligned}
$$

Among them, and for the width and height of the image, are the component images in pixel values.

A similar projection histogram can be obtained, in the direction and direction of the image component: and.

\subsection{Feature Extraction}

Projection histogram of the image is discrete sequence; sequence length depends on the width and height of the image [8]. Different image projection histogram processing, projection histogram will get different length. Based on the calculation of similarity of images, use the first three moment's projection histogram.

Central moment histogram shows the distribution histogram. On the first three moments of histogram, respectively:

$$
\begin{gathered}
M_{1}=\frac{1}{L} \sum_{i=1}^{L} P(i), \\
M_{2}=\sqrt{\frac{1}{L} \sum_{i=1}^{L}\left(P(i)-M_{1}\right)^{2}} \\
M_{3}=\sqrt[3]{\frac{1}{L} \sum_{i=1}^{L}\left(P(i)-M_{1}\right)^{3}} .
\end{gathered}
$$

Using HSI model, components are projected to the direction and the direction, get 4 projection histogram, 
i.e., and, respectively to calculate the first three moments of the 4 histograms, 12 central moment. The 12 center distance as features to describe images, the image can be represented by a 12 dimensional vector:

$$
\begin{aligned}
m= & \left(M_{1}^{H x}, M_{2}^{H x}, M_{3}^{H x}, M_{1}^{H y}, M_{2}^{H y}, M_{3}^{H y}, M_{1}^{I x}, M_{2}^{I x}, M_{3}^{I x},\right. \\
& \left.M_{1}^{I y}, M_{2}^{I y}, M_{3}^{I y}\right)
\end{aligned}
$$

Element represents a central moment's projection histogram in the direction of the image component, other elements in the vector meaning is similar.

\subsection{Similarity Calculation}

Let be a query image, is an image in the database, they are available for above 12 dimensions, respectively, and said:

$$
\begin{aligned}
q= & \left(Q_{1}^{H x}, Q_{2}^{H x}, Q_{3}^{H x}, Q_{1}^{H y}, Q_{2}^{H y}, Q_{3}^{H y}, Q_{1}^{I x}, Q_{2}^{I x}, Q_{3}^{I x},\right. \\
& \left.Q_{1}^{I y}, Q_{2}^{I y}, Q_{3}^{I y}\right) \\
d= & \left(D_{1}^{H x}, D_{2}^{H x}, D_{3}^{H x}, D_{1}^{H y}, D_{2}^{H y}, D_{3}^{H y}, D_{1}^{I x}, D_{2}^{I x}, D_{3}^{I x},\right. \\
& \left.D_{1}^{I y}, D_{2}^{I y}, D_{3}^{I y}\right)
\end{aligned}
$$

The image and the distance between the vector and is the distance between the weighted Euclidean distance to measure, as follows:

$$
\sqrt{W_{H} \sum_{i=1}^{3} \sum_{i=x, y}\left(Q_{i}^{H j}-D_{i}^{H j}\right)^{2}+W_{I} \sum_{i=1}^{3} \sum_{j=x, y}\left(Q_{i}^{I j}-D_{i}^{I j}\right)^{2}}
$$

Among them, as the weighted coefficients, is a group of experience, can be adjusted according to the test results. This paper retrieval system, $=0.7,=0.3$, because the component description ability and color visual approach, only components of projection in horizontal and vertical directions reflect the image shape information.

\section{Experimental Analysis}

In order to verify the statistical projection retrieval results, the author designed a set of experiments, a kind of image retrieval of the landscape, the number of images of 100 pieces, including image similar to figure 1 image has 10 beds, each query returns with the query image of 20 images most similar.

Figure 1 for the users to submit images, figure 2 to 20 results the global color histogram retrieval feedback method, figure 3 for statistical projection method to retrieve 20 results feedback to the system. According to figure 1, figure 2 and figure 3 analyses:

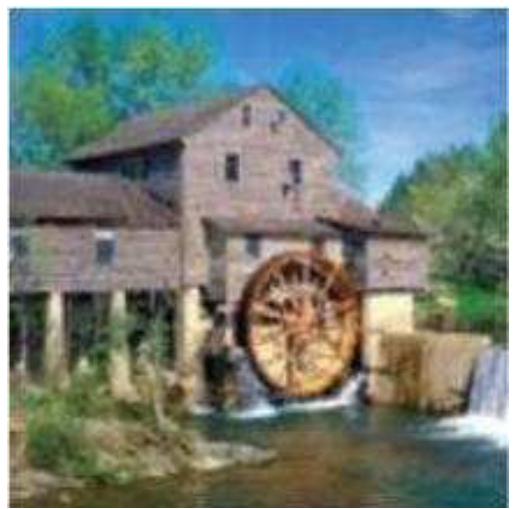

Fig. 1: users to submit images

1.due to the result according to the sorted result from small to large, from left to right, from top to bottom. In Fig. 2 and Fig. 3 image upper left corner is retrieved is the original.

2.Theory, because the system is used in extracting the principal value, projection histogram and weighted Euclidean distance, and in the weighted Euclidean distance, $=0.7,=0.3$, which makes the system on the component dependence. From the search results, we can be seen as the image submitted by users (Figure 1) at the top of the blue sky, the main color to blue. In the global color retrieval, image retrieval is the mainly blue. Search in statistical projection method results in image retrieval, it is also mainly blue, but with the global color histogram retrieval output have different results, because this system uses the projection of the main color and gray histogram projection, the main color histogram reflects the spatial distribution information of color, projection of the main gray histogram reflects the shape information of an image, but due to the use of the weighted Euclidean distance, $=0.7,=0.3$, weakening the image shape information, but with global histogram can not provide shape information.

\section{Conclusion}

This paper presents a statistical projection image retrieval based on color, can describe the spatial distribution information of image, but the shape of the spatial distribution of information that is not very precise, the method used in this paper to the redundant information in the image color filter, and can overcome the color histogram can not describe the spatial color distribution of defects. Retrieved using statistical projection method, the small amount of calculation, fast retrieval speed, can choose the characteristics and different weight according to the need of. How to integrate statistical projection method with other algorithms, improve the distribution to 


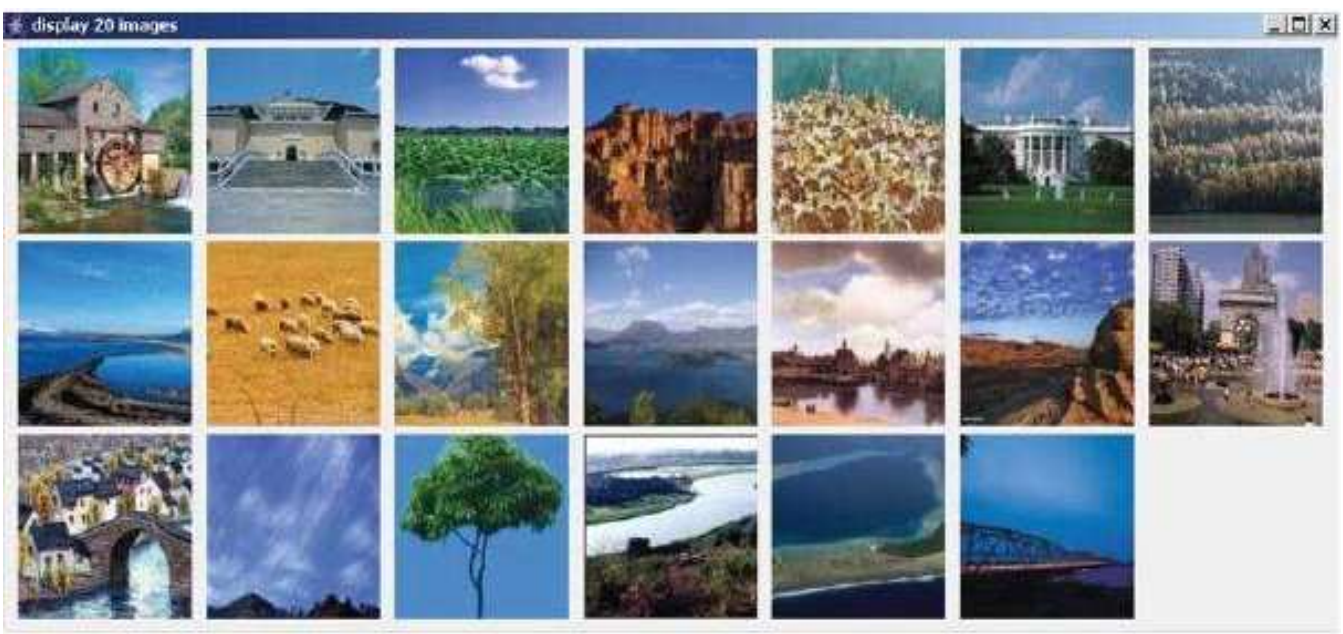

Fig. 2: the global color histogram comparison

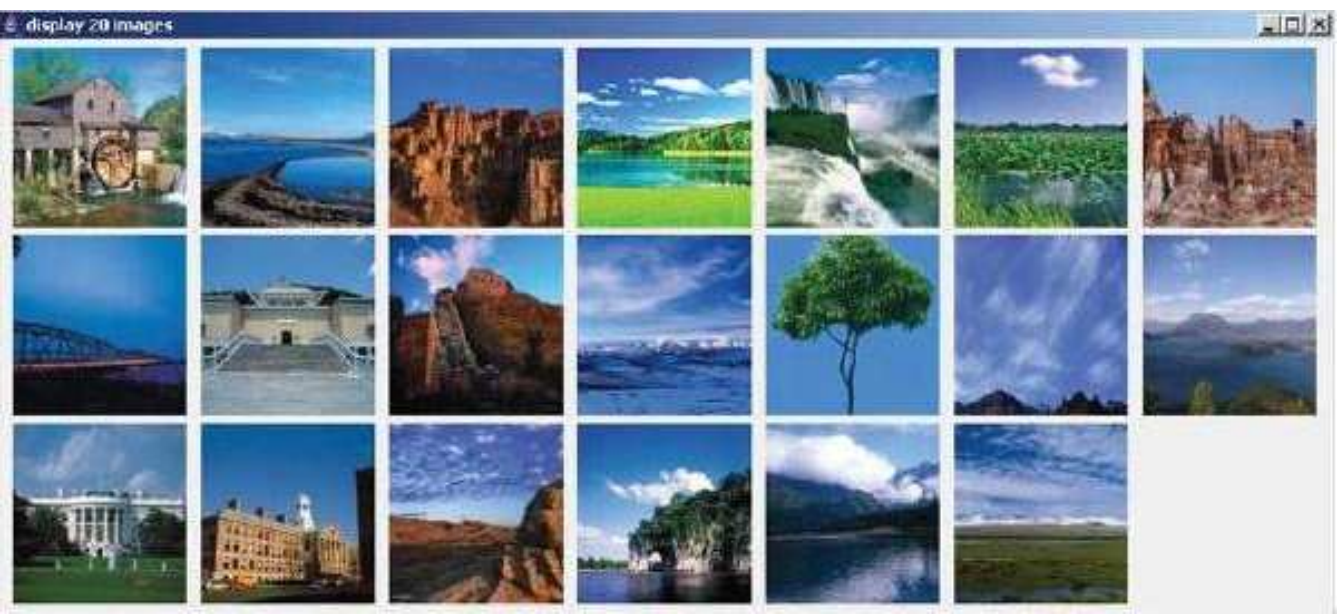

Fig. 3: using statistical projection search results

describe the shape information of space, is the next step of research content.

\section{Acknowledgement}

The work is supported by the fund of China Academy of Tropical Agricultural Sciences postdoctoral foundation.

\section{References}

[1] Chen Xinghua. The evolution of agricultural informationization and countermeasures $[\mathrm{J}]$. Science and technology management research, 437-03 (2009).

[2] Cao Yong. To speed up the agricultural informationization construction to promote agricultural modernization development [J]. Journal of Inner Mongolia science and technology and economy, 18, 63-64 (2009).
[3] Zhao chun. Henan province rural informatization way and strategy analysis $[\mathrm{J}]$. Journal of management informationization in China, 12, 81-83 (2009).

[4] Du Xulin Zhu Qin, Wen Huaiyu. New rural informationization present situation and development countermeasures [J]. Rural economy, 95-98 (2009).

[5] HUANGSH, SHEORANSK, WANGG. A review and analysis of supply chain operations reference (SCOR) model [J]. Supply Chain Management, 9, 23-29 (2004).

[6] CHOITY, DOOLEYKJ. Supply networks and complex adaptive systems: control versus emergence [J]. Journal of Operations Management, 19, 351- 366 (2001).

[7] Zhang Ailing. What time thinking about the construction of speed up agricultural informationization [J]. Journal of agricultural science and technology, 17-18 (2007).

[8] Yang Xiaorong. Thinking about China's rural informatization construction [J]. World agriculture, 19-21 (2008).

[9] Jiang Xiangdong. Strategy analysis of agricultural informatization development [J]. Journal of agricultural 
mechanization research, 32-35 (2005).

[10] Liu WanDai. Ma Xinming. Agricultural information technology development research in the construction of new socialist rural [J]. Agricultural science of Anhui, 2911-2912 (2006).

[11] Smith J R. Color for image retrieval [A]. Casteli VBergman L Deds. Image Databases-Search and Retrieval for Digital Imagery [C]. John Wiley \& Sons, (2002).

[12] Swain M J, Ballard D H. Color indexing [J]. International Journal of Computer Vision, 7, 11-32 (1997).

[13] Zhang Y J, Yao Y RHe Y. Color image segmentation based on HIS Model [J]. Heigh Technology Letters, 4, 28-31 (1998).

[14] Beeze-Yates RRibeiro-Neto B. Modern Information Retrieval [M]. Addison Wesley, (1999).

[15] V N Gudivada, V V Raghavan. Content based image retrieval systems [J]. IEEE Computer, 199528, 18-22.

[16] A YoshitakaT Ichikawa. A survey on content-based retrieval for multi-media databases [J]. IEEE Transaction on Konwledge and Data Enginneering, 11, 81-93 (1999).

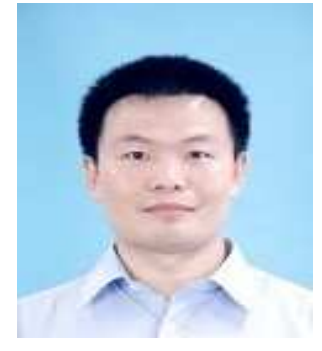

Xiaofei Zheng received Ph.D in College of Agriculture at Hainan University. $\mathrm{He}$ is Assistant Professor of the Institute of Tropical Agricultural Science and Technology Information of CATAS. His research interests are of agricultural economics, agricultural informatization, germplasm resources development, agricultural strategy \& policy, global agricultural development, etc. He has published two academic monographs "Economics Study of Wild Rice Germplasm in Hainan" and "Study on Multilevel Development for Agricultural Informatization in Hainan". He has published more than 20 research manuscripts in different scientific journals, such as China Informatization, China Tropical Agriculture, Agricultural Economics Research, Hubei Agricultural Science, Ecological Economy, China Study Journal of Malaya University, etc. And one article indexed by The Science Citation Index, three articles indexed by The Engineering Index.

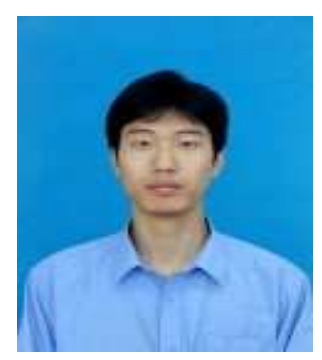

Gao Zhe received MEng in College of Information Science \& Technology at Hainan University. His research interests are of image processing, content-based image retrieval. $\mathrm{He}$ has published research article in Natural Science Journal of Hainan University.

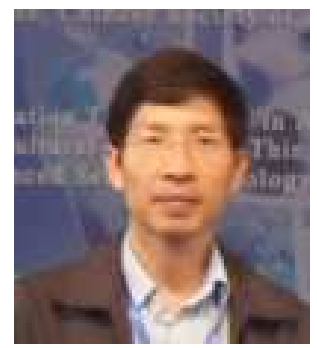

Wei Luo, Ph.D., Professor, Doctoral Supervisor, Deputy Director of the Institute of Tropical Agricultural Science and Technology Information of CATAS, has long been engaged in the research work of Tropical Agricultural informatization. 